\section{Concordância entre informações do Cartão da Gestante e do recordatório materno entre puérperas de uma cidade brasileira de médio porte}

\author{
Agreement between data from prenatal care cards \\ and maternal recall in a medium-sized Brazilian city \\ Correspondencia entre la información de la cartilla \\ de gestante y el diario materno entre puérperas de \\ una ciudad media brasileña
}

\section{${ }^{1}$ Faculdade de Medicina, Universidade Federal do Rio Grande, Rio Grande, Brasil. \\ Correspondência M. Zanchi \\ Programa de Pós-graduação em Ciências da Saúde, \\ Faculdade de Medicina, Universidade Federal do Rio Grande. \\ Rua Visconde de Paranagua 102, Rio Grande, RS 96200-190, Brasil. marizazanchi@hotmail.com}

\begin{abstract}
Prenatal care is a key indicator of the quality of health services. The current study aimed to evaluate the correlation between data from prenatal care cards and maternal recall in the city of Rio Grande, Rio Grande do Sul State, Brazil. The cross-sectional study included all mothers from 2010 who had received prenatal care. Interviews were conducted with a pre-coded questionnaire in the maternity hospital. Of the 2,288 mothers interviewed, 1,228 (53.7\%) had the prenatal care card with them and thus comprised the group for comparison. The analysis used kappa correlation and confidence interval. The variables six or more prenatal visits, clinical breast and gynecological examination, two blood tests, VDRL, HIV serology, urine test, and tetanus vaccination showed statistically significant differences between annotated and maternal recall data ( $p \leq$ 0.001). Adequacy of prenatal care based on the guidelines of the Program for Humanization of Prenatal Care (PHPN) was 23.9\% according to information provided by the patients and $4.4 \%$ according to information recorded on the prenatal cards $(p \leq 0.001)$. The prenatal care card showed underreporting, which limited the quality assessment of prenatal care.
\end{abstract}

Health Care Quality Indicators; Medical Audit; Prenatal Care; Health Evaluation
Mariza Zanchi 1

Carla Vitola Gonçalves 1

Juraci A. Cesar 1

Samuel de Carvalho Dumith ${ }^{1}$
A assistência pré-natal é um indicativo da qualidade dos serviços de saúde. Esse estudo objetivou avaliar a concordância entre os dados do pré-natal da memória materna e do cartão da gestante no Município do Rio Grande, Rio Grande do Sul, Brasil. Estudo transversal incluindo todas puérperas do ano de 2010 que realizaram pré-natal. As entrevistas e a coleta dos dados do cartão ocorreram nas maternidades. Das 2.288 puérperas entrevistadas, 1.228 (53,7\%) portavam o cartão da gestante, sendo a comparação realizada nesse grupo. A análise foi feita utilizandose o teste de concordância kappa e o intervalo de confiança. A realização de seis ou mais consultas de pré-natal, exame das mamas e ginecológico, dois exames de sangue, VDRL, anti-HIV, urina e a vacinação antitetânica demonstraram diferença estatística entre os dados referidos e anotados ( $p \leq 0,001)$. A adequação do pré-natal pelo índice do Programa de Humanização do PréNatal (PHPN) nos dados referidos foi de 23,9\% e de 4,4\% nos dados anotados ( $p \leq 0,001)$. Observamos um sub-registro no cartão da gestante, $o$ qual influenciou negativamente na avaliação da qualidade do pré-natal.

Indicadores de Qualidade em Assistência à Saúde; Auditoria Médica; Cuidado Pré-natal; Avaliação em Saúde 


\section{Introdução}

A assistência pré-natal adequada consiste em prevenir, em diagnosticar e em tratar eventos indesejáveis na gestação, no parto e no puerpério 1 . Essa atenção é fundamental à redução da morbimortalidade materna e infantil, portanto a qualidade desses cuidados está diretamente relacionada à saúde integral de mães e de conceptos 2,3,4. A mortalidade materna é um importante indicador da realidade social de um país 5 , e a mortalidade perinatal é um indicador sensível da qualidade da assistência obstétrica e neonatal e do impacto dos programas de prevenção 6 .

No Brasil, nas últimas décadas, surgiram políticas públicas direcionadas à saúde no ciclo gravídico-puerperal, por meio da implantação de programas e de ações que são importantes na organização dos serviços de saúde 7 . Dentre elas, o Programa de Humanização do Pré-Natal (PHPN), lançado pelo Ministério da Saúde em 2000 8, com a intenção de promover mudanças no cenário social. O PHPN regulamentou as atividades a serem desenvolvidas no pré-natal e preconizou critérios para avaliar a assistência prestada: a taxa de cobertura do programa, a realização de seis ou mais consultas de pré-natal, o início no primeiro trimestre de gestação, a realização de exames laboratoriais mínimos em dois momentos distintos da gravidez, a vacinação e a suplementação com sulfato ferroso.

A aplicação do PHPN para avaliar o processo da assistência pré-natal proporciona identificar o desempenho do serviço e evidencia a qualidade da assistência, sendo essa uma das condições para garantir a efetividade dos cuidados à gestante ${ }^{9}$. A investigação da qualidade pré-natal pode ser concretizada por intermédio de entrevista com as pacientes, da auditoria dos prontuários e da auditoria do Cartão da Gestante, contemplando os cuidados efetivamente oferecidos e recebidos na assistência 10,11 .

A auditoria médica avalia a qualidade da atenção refletida nos registros das histórias clínicas das pacientes. Os auditores podem revisar as fichas das pacientes atendidas ou os Cartões da Gestante, e avaliar se a assistência recebida ajusta-se aos critérios previamente definidos 12 . Considera-se que se algum procedimento ou exame complementar não foi registrado, provavelmente, ele não foi realizado $12,13,14,15,16$. Por isso, os registros médicos são muito importantes na prática clínica, influenciando decisivamente nos processos de cuidado ${ }^{12}$. No entanto, estudos que avaliem os dados por meio do registro dos procedimentos são pouco realizados por dificuldades tanto na portabilidade do cartão pelas gestantes, quanto no seu completo e correto preenchimento pelos profissionais de saúde 15,17 .

Vários estudos de avaliação da qualidade do pré-natal são realizados com os dados referidos pela mãe em entrevistas, que podem ser considerados mais rápidos e de menor custo, porém com limitação à validação dos dados, devido à compreensão e ao entendimento da pergunta pelas entrevistadas, que, ao desconhecerem eventuais termos médicos questionados, podem dar respostas incorretas ou, ainda, devido ao viés recordatório materno, visto que algumas mães podem ter esquecido ou omitido informações na entrevista $18,19,20,21,22,23$.

As pesquisas de qualidade em atenção de saúde são instrumentos geradores de evidências científicas necessárias para o conhecimento da situação atual do atendimento pré-natal prestado, contribuindo para fornecer subsídios aos gestores de saúde no planejamento e na implementação de medidas. O presente estudo propõe verificar a adequação do pré-natal segundo os critérios do PHPN, comparando os dados referidos da memória materna com os dados anotados do Cartão da Gestante.

\section{Materiais e métodos}

Esse estudo foi realizado no Município do Rio Grande, localizado na planície costeira sul, no Estado do Rio Grande do Sul, que possui cerca de 200 mil habitantes, com 95\% deles residindo em área urbana. Possui o segundo maior porto marítimo do Brasil, com destaque para o polo naval e indústria de fertilizantes. Segundo o Instituto Brasileiro Geografia e Estatística (IBGE) 24, o município tem renda per capita mensal de $\mathrm{R} \$ 1.528,00$, sendo o 4o PIB do estado e o Índice de Desenvolvimento Humano (IDH) é de 0,792. A sua rede de saúde é constituída por 32 unidades básicas de saúde (UBS), dois hospitais gerais e cinco ambulatórios. Pelo menos $99 \%$ dos partos ocorrem em nível hospitalar. O presente estudo entrevistou todas as puérperas nas duas únicas maternidades do município, sendo elas a do Hospital Universitário Dr. Miguel Riet Corrêa Jr. e a da Associação de Caridade Santa Casa do Rio Grande.

Esse estudo é parte de um estudo maior intitulado Estudo Perinatal no Município do Rio Grande - 2010, da Universidade Federal do Rio Grande (FURG). Esse estudo investigou o perfil epidemiológico das puérperas, a assistência ao pré-natal, parto e puerpério e as características dos recém-nascidos da cidade do Rio Grande.

Foram incluídos nesse estudo todos os nascimentos ocorridos nas maternidades do mu- 
nicípio entre 1o de janeiro e 31 de dezembro de 2010. Para fazer parte do estudo, as puérperas deveriam residir na área urbana ou rural do município e ter parto de recém-nascido com peso igual ou superior a 500 gramas, ou, no mínimo 22 semanas de idade gestacional. Foram excluídas as pacientes que não haviam realizado pelo menos uma consulta de pré-natal.

No período do estudo, ocorreram 2.464 partos de mulheres residentes em Rio Grande nas duas maternidades do município, dentre essas $107(4,3 \%)$ não realizaram o pré-natal e 69 (2,8\%) pacientes se recusaram a participar do estudo, das 2.288 puérperas entrevistadas, apenas 1.228 (53,7\%) apresentavam o Cartão da Gestante em mãos no momento da entrevista. Portanto, a comparação entre os dados da rotina pré-natal referidos pela paciente e os dados anotados do Cartão da Gestante, coletados do registro médico, foi realizada apenas neste grupo.

Dez entrevistadoras com no mínimo Ensino Médio completo foram treinadas e contratadas para atuar neste estudo. Este treinamento consistiu na leitura do questionário e do manual de instruções, na sua aplicação entre duplas e perante o grupo de entrevistadoras. O estudo piloto foi realizado no mês anterior ao início da coleta de dados nas duas maternidades da cidade com o objetivo de testar o questionário a ser utilizado bem como avaliar o tempo necessário à realização da entrevista. Cada entrevistadora realizou duas entrevistas com puérperas internadas nas maternidades.

A identificação das puérperas era feita através de informação obtida diretamente junto às mesmas e confirmada através do livro de registro de nascimentos de cada maternidade. Ao encontrar a puérpera explicava-se a ela sobre os objetivos do estudo e a convidava para dele participar. Havendo concordância, um termo de consentimento era assinado por ela ou pelo seu representante legal autorizando a realização da entrevista.

As informações desse estudo foram coletadas por meio de questionário único pré-codificado, com entrevista às puérperas, nas duas únicas maternidades entre 12 e 48 horas após o parto. Esse questionário investigou características sociodemográficas (idade materna, cor da pele, escolaridade, se tinha companheiro, renda familiar em salário mínimo e quartil); história reprodutiva (número de gestações); assistência recebida durante a gestação (local do pré-natal, trimestre do início do pré-natal, número de consultas de pré-natal, aferição da pressão sanguínea e altura uterina, peso materno, exame das mamas, exame ginecológico, exames laboratoriais) e imunização antitetânica. No final da entrevista, era solicitado o Cartão da Gestante para a anotação de dados referentes à realização das rotinas da atenção pré-natal preconizadas pelo PHPN. Quando não houvesse nenhum registro a respeito de um procedimento no cartão, esse era considerado não realizado.

O controle de qualidade constou de repetição de cerca de $5 \%$ das entrevistas, sendo estas realizadas, na maioria das vezes, por telefone. Para as variáveis avaliadas, o índice kappa variou de 0,63 a 0,78 , sendo que os maiores índices obtidos foram para as variáveis "tipo de parto", "idade" e "cor da pele", enquanto os menores foram para "idade de início das consultas de pré-natal" e "renda familiar". A concordância observada foi, portanto, de moderada a satisfatória.

Para a avaliação da adequação da assistência pré-natal foram utilizados o índice proposto por Kessner et al. ${ }^{25}$, que descreve o pré-natal como adequado quando inicia no primeiro trimestre da gestação e tem seis ou mais consultas, o índice de Silveira et al. 13, que considera o pré-natal adequado quando inicia no primeiro trimestre, seis ou mais consultas e a realização de, no mínimo, dois exames qualitativos de urina, hemoglobina e sorologia para sífilis (VDRL), e o índice do PHPN 8, que define como pré-natal adequado o início no primeiro trimestre, seis ou mais consultas, a realização de procedimentos clínicoobstétricos (peso, aferição da pressão sanguínea arterial, medida da altura uterina, exame de mamas e ginecológico), dois exames qualitativos de urina, hemoglobina, glicemia, VDRL e anti-HIV, e a vacinação antitetânica.

A digitação dos questionários foi duplamente realizada. Os dados foram digitados em banco construído no Epi Info 6.04 (Centers for Disease Control and Prevention, Atlanta, Estados Unidos). Em seguida, realizou-se análise de consistência, que consistiu na criação e na categorização de variáveis e verificação de frequências realizadas por intermédio do programa SPSS 19.0 (SPSS Inc., Chicago, Estados Unidos). As questões abertas foram codificadas posteriormente, categorizando segundo as respostas mais frequentes. A análise utilizou os softwares Epi Info 6.04, SPSS 19.0 e Stata 11.0 (Stata Corp., College Station, Estados Unidos) onde foram calculados os intervalos de 95\% de confiança (IC95\%), o valor de p pelo teste de McNemar, a concordância observada e o kappa 26, teste de concordância que corrige $o$ acaso. A concordância pelo kappa foi considerada ruim $(\leq 0,20)$, considerável $(0,21$ $0,40)$, moderada $(0,41-0,60)$, substancial $(0,61-$ $0,80)$ e excelente $(0,81-1,00)$.

O protocolo de pesquisa foi submetido e aprovado pelo Comitê de Ética em Pesquisa na Área da Saúde (CEPAS), na FURG, parecer no 117/2009, e pelo CEPAS da Associação de Carida- 
de da Santa Casa de Misericórdia do Rio Grande, no 09/2009.

\section{Resultados}

Entre as 1.228 mulheres portadoras do Cartão da Gestante na maternidade, observou-se que $28,1 \%$ tinham entre 20 e 24 anos de idade, sendo que $20,4 \%$ das gestações eram em mulheres com idade menor ou igual a 19 anos. Além disso, $67,8 \%$ das mulheres eram brancas, $49,8 \%$ delas tinham até oito anos de escolaridade e $84,5 \%$ tinham companheiro no momento da pesquisa. Quanto aos dados socioeconômicos, 77,7\% das mulheres tinham renda familiar menor que três salários mínimos. Nos dados obstétricos, constatou-se que $45,2 \%$ eram primigestas, e $75,6 \%$ delas realizaram pré-natal nas UBS do município (Tabela 1).

Na Tabela 2, observou-se a comparação entre os dados referidos pela gestante na entrevista e os dados anotados no cartão da gestante. Nas entrevistas, $80 \%$ delas referiram ter realizado seis ou mais consultas de pré-natal; informação, entretanto, que só estava anotada em $74 \%$ dos cartões dessas, apresentando kappa de 0,76 (IC95\%: 0,71 0,80 ). A média de consultas realizadas no prénatal referida foi de oito e de sete anotadas no cartão. O início do pré-natal no primeiro trimestre demonstrou diferença estatística significante entre o dado referido e o dado anotado, apresentando kappa de 0,93 (IC95\%: 0,90; 0,95).

Quanto ao exame clínico ginecológico, pôdese observar que a aferição da pressão sanguínea arterial, a pesagem da gestante e a medida da altura uterina não apresentaram diferença estatística entre os dados referidos e os dados anotados, entretanto o exame das mamas e o exame ginecológico apresentaram importantes diferenças entre os grupos. O exame das mamas foi referido por $57,9 \%$ das pacientes e anotado em $32,9 \%$ dos cartões com kappa de 0,36 (IC95\%: 0,31; 0,40). Já o exame ginecológico teve sua realização referida por $65,3 \%$ das mulheres, mas foi anotado apenas em 33,7\% dos Cartões da Gestante com kappa de 0,20 (IC95\%: 0,15; 0,24). Entre os procedimentos clínicos avaliados no estudo, o exame da mama e o exame ginecológico apresentaram a pior concordância observada com valor kappa de 0,36 e 0,20 respectivamente.

Ao avaliarem-se os exames laboratoriais, observaram-se diferenças entre os dados referidos e os dados anotados no Cartão da Gestante, a realização de dois exames de sangue foi referida por $81,6 \%$ das mulheres, mas anotada em $42,2 \%$ nos Cartões da Gestante (kappa de 0,27). A sorologia de sífilis (VDRL) foi referida por $56,7 \%$ das pacientes e anotada em 35,9\% dos Cartões da Gestante (kappa de 0,31). O anti-HIV foi referido por $63,7 \%$ delas e anotado em $38,2 \%$ dos cartões (kappa de 0,33). O exame qualitativo de urina foi referido por $76,2 \%$ das pacientes e anotado em $44,1 \%$ dos cartões (kappa de 0,30 ). A vacinação antitetânica atualizada foi referida em $81,3 \%$, no entanto foi registrada em $50,1 \%$ dos cartões. O valor do kappa tanto para os exames laboratoriais como para a vacinação mostra uma ligeira concordância entre os valores referidos e os anotados.

Ao avaliar-se a adequação do pré-natal, constatou-se que o índice de Kessner et al. 25, apesar de demonstrar diferença estatística significante, teve seus índices muito próximos entre os dados referidos, 69,8\% (IC95\%: 67,2; 72,3), e os dados anotados no Cartão da Gestante, 64,6\% (IC95\%: 61,9; 67,3). Já o índice de Silveira et al. 13, que contempla também alguns exames laboratoriais, demonstrou maior diferença estatística entre os dados referidos, 70,7\% (IC95\%: 68,1; 73,3 ), e os dados anotados, $24,1 \%$ (IC95\%: 21,6 26,5). E, por último, quando se avaliaram os critérios do PHPN, a adequação do pré-natal nos dados referidos foi de 23,9\% (IC95\%: 21,4; 26,3) e de apenas 4,4\% (IC95\%: 3,2; 5,6) nos dados anotados no Cartão da Gestante kappa de 0,01 (IC95\%: -0,03; 0,05).

\section{Discussão}

Segundo recomendações do Ministério da Saúde, toda gestante deve ter assegurada assistência digna de qualidade no decorrer da gestação, do parto e do puerpério. Estudos com informações epidemiológicas são necessários para avaliar a efetividade do cuidado prestado e essenciais para o direcionamento de ações dos serviços de saúde.

O Cartão da Gestante é um documento de registro e deve conter dados referentes a todos os procedimentos realizados no acompanhamento da gestação 12,13,14,15,16. Segundo o Ministério da Saúde, é obrigatório o preenchimento do cartão na primeira consulta de pré-natal e ele deve ser entregue à paciente, que precisa portá-lo sempre, sendo fundamental para sua referência e contra-referência 8 . Uma das limitações deste estudo é que apenas $53,7 \%$ das pacientes portavam o Cartão da Gestante no momento da entrevista realizada na maternidade, acredita-se que alguns cartões possam ter ficado no centro obstétrico na admissão da paciente. Como o cartão da gestante é o documento que contém o histórico da gestação, os profissionais da saúde deveriam devolvêlo à paciente. Além disso, o médico pré-natalista 
Tabela 1

Características sociodemográficas da amostra das 1.228 puérperas que portavam o Cartão da Gestante na maternidade. Rio Grande, Sul do Brasil, 2010

\begin{tabular}{|c|c|c|}
\hline Variáveis & Frequência & $\%$ \\
\hline \multicolumn{3}{|l|}{ Idade (anos) } \\
\hline$\leq 19$ & 251 & 20,4 \\
\hline $20-24$ & 345 & 28,1 \\
\hline $25-29$ & 313 & 25,5 \\
\hline$\geq 30$ & 319 & 26 \\
\hline \multicolumn{3}{|l|}{ Cor da pele } \\
\hline Branca & 833 & 67,8 \\
\hline Não branca & 395 & 32,2 \\
\hline \multicolumn{3}{|l|}{ Companheiro } \\
\hline Com marido ou companheiro & 1.037 & 84,5 \\
\hline Sem marido ou companheiro & 191 & 15,5 \\
\hline \multicolumn{3}{|l|}{ Escolaridade (anos completos) } \\
\hline $0-8$ & 612 & 49,8 \\
\hline $9-11$ & 516 & 42,0 \\
\hline 12 ou mais & 100 & 8,2 \\
\hline \multicolumn{3}{|l|}{ Renda (salários mínimos) } \\
\hline$\leq 1$ & 284 & 23,1 \\
\hline $1-3$ & 670 & 54,6 \\
\hline$>3$ & 274 & 22,3 \\
\hline \multicolumn{3}{|l|}{ Número de gestações } \\
\hline Primigesta & 555 & 45,2 \\
\hline Secundigesta & 347 & 28,3 \\
\hline Multigesta & 326 & 26,5 \\
\hline \multicolumn{3}{|l|}{ Local do pré-natal } \\
\hline Posto de saúde & 928 & 75,6 \\
\hline Ambulatório de hospital universitário ou ambulatório público & 245 & 20,0 \\
\hline Médico conveniado ou particular & 55 & 4,4 \\
\hline
\end{tabular}

poderia ressaltar para a paciente a importância de portar o cartão, na sua consulta puerperal.

Muitas pesquisas que avaliam a assistência pré-natal são realizadas com informações a partir de relatos. Esses estudos são rápidos e de baixo custo, porém uma de suas limitações diz respeito à validação de alguns dados colhidos por entrevistas, principalmente aqueles que dependem da memória e da boa vontade da informante 18,19,20,21,22,23. Por conseguinte, há a possibilidade de viés recordatório, visto que algumas mães podem ter esquecido ou omitido dados. Estudos que utilizam a auditoria do cartão da gestante podem ser considerados mais fidedignos para refletir a qualidade da atenção prestada 27. Entretanto, esses estudos mostram a baixa qualidade no preenchimento de dados pelos profissionais de saúde, e esse sub-registro constitui uma limitação, pois a ausência de re- gistros pressupõe a não realização dos procedimentos 12,13,14,15,16,17.

No presente estudo, observou-se que, no grupo dos dados referidos, $80 \%$ das puérperas relataram ter realizado seis ou mais consultas de pré-natal, com uma média de oito consultas. No entanto, no grupo dos dados anotados no Cartão da Gestante, apenas $74 \%$ das puérperas tinham registro de ter realizado seis ou mais consultas, com uma média de 7,4 consultas. Estudo realizado por Santos Neto et al. 16, comparando a quantidade de consultas pré-natais informadas pelas puérperas com as coletadas nos Cartões da Gestante, mostrou uma tendência a superestimação do número de consultas pelas puérperas em relação aos cartões. Estudos realizados com dados coletados por entrevista mostraram uma prevalência de realização de seis consultas ou mais no pré-natal de $67 \%$ a $76,3 \% 20,28,29$. Observou-se 
Tabela 2

Avaliação da concordância entre os dados referidos pela puérpera e dos dados anotados do Cartão da Gestante. Rio Grande, Sul do Brasil, 2010

\begin{tabular}{|c|c|c|c|c|c|c|}
\hline Variável & $\mathbf{n}$ & $\begin{array}{l}\text { Referido } \\
\text { \% (IC95\%) }\end{array}$ & $\begin{array}{l}\text { Carteira } \\
\% \text { (IC95\%) }\end{array}$ & $\begin{array}{l}\text { Valor de p } \\
\text { (teste de } \\
\text { McNemar) }\end{array}$ & $\begin{array}{l}\text { Concordância } \\
\text { observada (\%) }\end{array}$ & Карра (IC95\%) \\
\hline Ter 6 ou mais consultas & 1.217 & $80,0(77,7 ; 82,3)$ & $74,0(71,6 ; 76,5)$ & $<0,001$ & 91,3 & $0,76(0,71 ; 0,80)$ \\
\hline Início do pré-natal no 1o trimestre & 1.228 & $78,4(76,1 ; 80,7)$ & $75,9(76,4 ; 78,3)$ & $<0,001$ & 97,5 & $0,93(0,90 ; 0,95)$ \\
\hline Medida da pressão arterial & 1.228 & $98,5(97,9 ; 99,2)$ & $97,9(97,1 ; 98,7)$ & 0,16 & 97,9 & $0,40(0,21 ; 0,54)$ \\
\hline Medida do peso & 1.228 & $99,4(99,0 ; 99,9)$ & $99,5(99,1 ; 99,9)$ & 1,00 & 94,3 & $0,46(0,12 ; 0,80)$ \\
\hline Medida da altura intrauterina & 1.228 & $99,4(99,0 ; 99,9)$ & $98,9(98,3 ; 99,5)$ & 0,19 & 98,3 & $-0,01(-0,01 ; 0,00)$ \\
\hline Exame das mamas & 1.228 & $57,9(55,1 ; 60,7)$ & $32,9(30,3 ; 35,5)$ & $<0,001$ & 66,2 & $0,36(0,31 ; 0,40)$ \\
\hline Exame ginecológico & 1.227 & $65,3(62,6 ; 68,0)$ & $33,7(31,1 ; 36,4)$ & $<0,001$ & 55,8 & $0,20(0,15 ; 0,24)$ \\
\hline 2 exames de sangue & 1.205 & $81,6(79,4 ; 83,8)$ & $42,2(39,5 ; 45,0)$ & $<0,001$ & 59,7 & $0,27(0,23 ; 0,30)$ \\
\hline 2 exames de VDRL & 1.206 & $56,7(53,9 ; 59,5)$ & $35,9(33,2 ; 38,6)$ & $<0,001$ & 63,9 & $0,31(0,26 ; 0,35)$ \\
\hline 2 exames de HIV & 1.216 & $63,7(60,9 ; 66,4)$ & $38,2(35,4 ; 40,9)$ & $<0,001$ & 64,3 & $0,33(0,28 ; 0,38)$ \\
\hline 2 exames qualitativos de urina (EQU) & 1.211 & $76,2(73,8 ; 78,6)$ & $44,1(41,3 ; 46,9)$ & $<0,001$ & 62,9 & $0,30(0,26 ; 0,34)$ \\
\hline Vacina antitetânica & 1.211 & $81,3(79,1 ; 83,5)$ & $50,1(47,3 ; 59,4)$ & $<0,001$ & 61,9 & $0,24(0,19 ; 0,28)$ \\
\hline Kessner et al. 25 & 1.217 & $69,8(67,2 ; 72,3)$ & $64,6(61,9 ; 67,3)$ & $<0,001$ & 93,7 & $0,86(0,83 ; 0,89)$ \\
\hline Silveira et al. 13 & 1.185 & $70,7(68,1 ; 73,3)$ & $24,1(21,6 ; 26,5)$ & $<0,001$ & 53,0 & $0,23(0,20 ; 0,26)$ \\
\hline PHPN & 1.153 & $23,9(21,4 ; 26,3)$ & $4,4(3,2 ; 5,6)$ & $<0,001$ & 74,0 & $0,01(-0,03 ; 0,05)$ \\
\hline
\end{tabular}

PHPN: Programa de Humanização do Pré-Natal.

Kessner et al. 25 adequado: início no primeiro trimestre e seis consultas ou mais; Silveira et al. 13 adequado: início no primeiro trimestre, seis consultas ou mais, dois exames de hemoglobina e VDRL, e dois exames de urina; PHPN adequado: início no primeiro trimestre, seis consultas ou mais, medida pressão da sanguínea arterial, pesagem, medida da altura uterina, exame das mamas, exame ginecológico, dois exames de sangue, HIV, VDRL e urina e VAT.

que, nos estudos de auditoria, a realização de seis ou mais consultas apresentou taxa de $63 \%$ e $64,9 \% 13,30$. Quanto ao início do pré-natal, houve diferença estatística entre os dados referidos e os dados anotados. Várias publicações mostram uma prevalência de início do pré-natal no primeiro trimestre entre $71,9 \%$ a $89,9 \% 4,20,29,30$ nos dados referidos e de $70,3 \%$ a $74,4 \% 31,32$ nos dados anotados do cartão, ou no prontuário médico. Portanto, a diferença estatística encontrada entre esses grupos pode ser explicada por duas teorias: ou temos uma subanotação no Cartão da Gestante, ou as mães, por desejo de se mostrarem cuidadosas, superestimam a realização das consultas e o início do pré-natal. Além disso, pudemos observar que a concordância entre os dados foi substancial e quanto ao número de consultas esse foi classificado como excelente.

Sobre os resultados do exame clínico ginecológico, observou-se concordância entre os dados de aferição da pressão arterial, da pesagem e da medida da altura uterina. A coerência entre o relato e o registro de exames considerados simples e rápidos e que, habitualmente, são realizados pela equipe de enfermagem, também foi observada em outros estudos 19,33,34. No entanto, procedimentos essenciais no cuidado pré-natal, como o exame de mamas e o exame ginecológico, que demandam maior tempo de consulta e habilidade, além de serem pouco realizados, apresentaram diferença estatística entre o dado referido e o anotado. A realização do exame das mamas foi referida por $57,9 \%$ das pacientes e registrada em $32,9 \%$ dos cartões. Quanto ao exame ginecológico, $65,3 \%$ das pacientes referiram ter realizado, no entanto o registro foi encontrado em 33,7\% dos cartões. Essa diferença também foi encontrada em estudo realizado em Recife, onde o exame de mamas foi referido por $74,4 \%$ das puérperas e anotado em $27,1 \%$ dos cartões, já o exame de Papanicolaou foi referido por $77,9 \%$ das mulheres e anotado em $29,4 \%$ dos cartões 35 . Acredita-se que a disparidade desses dados deva-se ao sub-registro desses, uma vez que são exames de difícil esquecimento pela sua magnitude íntima. Outro fato que pode contribuir para a ausência de registro dos exames no cartão da gestante é a sua localização, de forma pouco valorizada e de difícil visualização.

Em relação à realização dos exames laboratoriais preconizados pelo PHPN, em dois momentos, observou-se diferença estatística ( $\mathrm{p} \leq 0,001)$ entre os dados referidos e os dados anotados para as seguintes variáveis: exames de sangue, 
VDRL, anti-HIV e exame qualitativo de urina. A rotina completa de exames foi relatada em média duas vezes mais do que registrada. Estudo realizado com 262 puérperas em Recife (Pernambuco), comparando os dados anotados do Cartão da Gestante com as informações colhidas junto às mulheres, demonstrou discrepância, uma vez que elas informaram terem sido submetidas aos exames laboratoriais com maior periodicidade do que o registrado no Cartão da Gestante 35. No Rio de Janeiro, estudo com 2.353 gestantes, com entrevistas e com dados do Cartão da Gestante, ressaltou que apenas um terço delas tinha registro dos exames da segunda rotina ${ }^{33}$. Acredita-se que possa haver a supervalorização do relato materno sobre a realização dos exames laboratoriais, principalmente dos exames de sangue e VDRL. No entanto, para realizar o anti-HIV, pressupõese que o aconselhamento pré e pós-coleta do exame torna mais difícil o seu esquecimento, ou mesmo um possível viés de memória. Da mesma forma, pensa-se ser difícil a paciente não lembrar quantas coletas de urina realizou.

Considerando a imunização antitetânica, o presente estudo encontrou uma cobertura vacinal referida de $81,3 \%$, porém somente em $50,1 \%$ dos cartões apresentavam esse dado anotado. De acordo com estudos anteriores, a cobertura vacinal por relato da paciente foi entre $64 \%$ a $91 \%$ 4,20,23. Estudos que usaram os dados do Cartão da Gestante mostraram uma cobertura vacinal, entre $54,2 \%$ e $83 \% 32,33,34$. Salienta-se que, em nosso município, a vacinação é realizada por profissional desvinculado do atendimento prénatal e em local e dias diferentes da consulta, podendo a gestante esquecer a portabilidade do Cartão da Gestante quando procura a vacinação. Outra hipótese seria de a gestante levar o Cartão de Vacinação para o pré-natalista que confere a vacinação atualizada, mas não registra esse dado no Cartão da Gestante.

No presente estudo, avaliou-se a adequação do cuidado pré-natal por meio dos indicadores de Kessner et al. 25, Silveira et al. 13 e PHPN. Todos esses índices apresentaram diferença estatística significante entre os dados referidos e anotados. Quando avaliou-se o índice de Kessner et al. 25, que usa apenas início do pré-natal e o número de consultas, notou-se uma diferença de $69,8 \%$ de adequação nos dados referidos, para $64,6 \%$ de adequação nos dados anotados. No entanto, observou-se maior diferença quando se associou ao índice os exames laboratoriais. Ao avaliar-se o índice de Silveira et al. 13, encontrou-se uma adequação de pré-natal de $70,7 \%$ para os dados referidos e de $24,1 \%$ para os dados anotados. Os piores resultados foram obtidos ao acrescentarem-se os procedimentos clínico ginecológicos, laboratoriais e a imunização, preconizados no PHPN, a adequação passou de 23,9\% nos dados referidos para $4,4 \%$ nos dados anotados. Esses achados são semelhantes aos verificados em estudos anteriores de qualidade de pré-natal, cujos percentuais variam de $26,8 \%$, a $36,7 \% 20,36$ nos dados referidos e de $0 \%$ a $7 \%$ nos dados anotados 23,32,33,34. Observou-se que a associação de um número maior de condutas pré-natais na avaliação dos índices de qualidade diminuiu a concordância entre eles, pois a concordância passou de 93,7\% com kappa de 0,86 no índice de Kessner et al. 25 , para concordância de $53 \%$ com kappa de 0,23 no índice de Silveira et al. 13 e concordância observada de $74 \%$ com kappa de 0,01 para o índice do PHPN.

Portanto não temos como saber de que lado está a inconsistência (se no registro ou no relato). No artigo todo, se coloca o relato como padrão-ouro, mas conforme relata o revisor, pode haver um erro no relato também. Então, devemos incluir este ponto como uma possível limitação do estudo. Quanto aos fatores associados à inconsistência cartão x autorrelato, com certeza acrescentaria bastante. Mas como a inconsistência varia de acordo com o indicador que está sendo visto, precisaríamos de um artigo para apresentar estes resultados, o que poderá ser feito futuramente.

Apesar de não termos como afirmar se a inconsistência dos dados está no relato ou no registro destes, com o presente estudo, pode-se concluir que além da subanotação do Cartão da Gestante, pode ter ocorrido a superestima das puérperas em relação a realização dos procedimentos preconizados. Em relação ao início do pré-natal, o número de consultas e a realização do exame de sangue, a superestima pela puérpera parece ser mais plausível. No entanto, para os exames de mamas e ginecológico, HIV e urina, acreditase ser mais frequente a subnotificação no Cartão da Gestante em relação ao que foi efetivamente realizado. Nesse estudo, o sub-registro influenciou negativamente na avaliação da qualidade do pré-natal. Por fim, é importante lembrar que a ausência de informações no Cartão da Gestante acarreta prejuízos na intercomunicação entre as diversas instâncias envolvidas na assistência ao pré-natal, ao parto e ao puerpério, por desconhecimento do real acompanhamento do cuidado prestado. Acrescenta-se, ainda, o desperdício de recursos, pois o não registro dos exames demanda uma nova realização desses no momento do parto, o que gera maior custo aos serviços de saúde. Sugere-se, assim, a sensibilização e a capacitação dos profissionais da saúde envolvidos na assistência pré-natal, no sentido da adesão às práticas baseadas em evidências científicas e 
registro completo e confiável do Cartão da Gestante. Uma medida que poderia ser adotada pelos serviços de saúde é a disponibilização de um maior tempo de consulta para a primeira e se- gunda consultas, já que nestes encontros são realizados o preenchimento do Cartão da Gestante, a realização do exame clínico-ginecológico, a solicitação e a verificação dos exames.

\section{Resumen}

La asistencia prenatal es un indicativo de la calidad de los servicios de salud. Este estudio tuvo por objetivo evaluar la correspondencia entre los datos prenatales del diario materno y de la Cartilla de Gestante en el municipio de Río Grande, Rio Grande do Sul, Brasil. Es un estudio transversal incluyendo a todas las puérperas del año 2010 que recibieron asistencia prenatal. Las entrevistas y la recogida de los datos de la cartilla se produjeron en las maternidades. De las 2.288 puérperas entrevistadas, 1.228 (53,7\%) llevaban la cartilla de gestante, realizándose la comparación en ese grupo. El análisis se ejecutó utilizándose el test de concordancia kappa y el intervalo de confianza. La realización de seis o más consultas de prenatal, examen de mamas y ginecológico, de los exámenes de sangre, VDRL, anti$V I H$, orina y la vacunación antitetánica demostraron una diferencia estadística entre los datos proporciona dos y los anotados ( $p \leq 0,001)$. La adecuación del servicio prenatal por el índice del Programa de Humanización de Prenatal (PHPN) en los datos recogidos fue de un 23,9\% y de un 4,4\% en los datos anotados ( $p \leq$ 0,001). Observamos un subregistro en la Cartilla de la Gestante, el cual influenció negativamente en la evaluación de la calidad de prenatal.

Indicadores de Calidad de la Atención de Salud; Auditoria Médica; Atención Prenatal; Evaluación de Salud

\section{Colaboradores}

M. Zanchi colaborou na elaboração do projeto e na redação do artigo. C. V. Gonçalves participou da elaboração do projeto, análise dos dados e redação do artigo. S. C. Dumith contribuiu na análise dos dados e revisão do artigo. J. A. Cesar colaborou na elaboração do projeto, supervisão da coleta e entrada dos dados e revisão do artigo.

\section{Agradecimentos}

Ao CNPq pelo financiamento. 


\section{Referências}

1. Uchoa JL, Sales AAR, Joventino ES, Ximenes LB. Indicadores de qualidade da assistência ao prénatal: realidade de gestantes atendidas em unidade de saúde de família. Rev Enferm UFPE on line 2010; 4:209-17.

2. World Health Organization. What is the effectiveness of antenatal care? Copenhagen: WHO Regional Office for Europe; 2005.

3. Ministério da Saúde. Programa de assistência integral à saúde da mulher. Brasília: Ministério da Saúde; 1983.

4. Victora CG, Aquino EML, Leal MC, Monteiro CA, Barros FC, Szwarcwald CL. Maternal and child health in Brazil: progress and challenges. Lancet $2011 ; 377: 1863-76$

5. Barros FC, Matijasevich A, Requejo JH, Giugliane E, Monteiro CA, Barros AJD, et al. Recent trends in maternal, newborn, and child health in Brazil: progress toward Millennium Development Goals 4 and 5. Am J Public Health 2010; 100:1877-89.

6. Lansky S, França E, Leal MC. Mortalidade perinatal e evitabilidade: revisão da literatura. Rev Saúde Pública 2002; 36:759-72.

7. Paim J, Travassos C, Almeida C, Bahia L, Macinko J. The Brazilian Health System: history, advances, and challenges. Lancet 2011; 377:1778-97.

8. Ministério da Saúde. Pré-natal e puerpério: atenção qualificada e humanizada. Brasília: Ministério da Saúde; 2005.

9. Nagahama EEI, Santiago SM. O cuidado pré-natal em hospital universitário: uma avaliação de processo. Cad Saúde Pública 2006; 22:173-9.

10. Reis EJFB, Santos FP, Campos FE, Acúrcio FA, Leite MTT, Leite MLC, et al. Avaliação da qualidade dos serviços de saúde: notas bibliográficas. Cad Saúde Pública 1990; 6:50-61.

11. Donabedian A. Promoting quality through evaluating the process of patient care. Med Care 1968; 6:181-202.

12. Dias-da-Costa JS, Madeira ACC, Luz RM, Britto MAP. Auditoria médica: programa de pré-natal em posto de saúde na região Sul do Brasil. Rev Saúde Pública 2000; 34:329-36.

13. Silveira DS, Santos IS, Dias-da-Costa JS. Atenção pré-natal na rede básica: uma avaliação da estrutura e do processo. Cad Saúde Pública 2001; 17:131-9.

14. Coutinho T, Teixeira MTB, Dain S, Sayd JD, Coutinho LM. Adequação do processo de assistência pré-natal entre as usuárias do Sistema Único de Saúde em Juiz de Fora, MG. Rev Bras Ginecol Obstet $2003 ; 25: 717-24$.

15. Coutinho T, Monteiro MFG, Sayd JD, Teixeira MTB, Coutinho CM, Coutinho LM. Monitoring the prenatal care process among users of the Unified Health Care System in a city of the Brazilian Southeast. Rev Bras Ginecol Obstet 2010; 32:563-9.

16. Santos Neto ET, Leal MC, Oliveira AE, Zandonade E, Gama SGN. Concordância entre informações do cartão da gestante e da memória materna sobre assistência pré-natal. Cad Saúde Pública 2012; $28: 256-66$
17. Koffman MD, Bonadio IC. Avaliação da atenção PN em uma instituição filantrópica da cidade de São Paulo. Rev Bras Saúde Matern Infant 2005; 5:523-32.

18. Cesar JA, Mano PS, Carlotto K, González-Chica DA, Mendoza-Sassi RA. Público versus privado: avaliando a assistência à gestação e ao parto no extremo sul do Brasil. Rev Bras Saúde Matern Infant 2011; 11:257-63.

19. Neumann NA, Tanaka OU, Victora CG, Cesar JA. Qualidade e equidade da atenção ao pré-natal e ao parto em Criciúma, Santa Catarina, Sul do Brasil. Rev Bras Epidemiol 2003; 6:307-18.

20. Gonçalves CV, César JA, Mendoza-Sassi RA. Qualidade e equidade na assistência à gestante: um estudo de base populacional no Sul do Brasil. Cad Saúde Pública 2009; 25:2507-16.

21. Victora CG, Matijasevich A, Silveira MF, Santos IS Barros AJD, Barros FC. Socio-economic and ethnic group inequities in antenatal care quality in the public and private sector in Brazil. Health Policy Plan 2010; 25:253-61.

22. Carvalho DS, Novaes HMD. Avaliação da implantação de programa de atenção pré-natal no Município de Curitiba, Paraná, Brasil: estudo em coorte de primigestas. Cad Saúde Pública 2004; 20 Suppl 2:S220-30.

23. Anversa ETR, Bastos GAN, Nunes LN, Dal Pizzol TS. Qualidade do processo de assistência pré-natal: unidades básicas de saúde e unidades de Estratégia Saúde de Família em município no Sul do Brasil. Cad Saúde Pública 2012; 28:789-800.

24. Instituto Brasileiro de Geografia e Estatística. Indicadores sociodemográficos e de saúde no Brasil, 2009. Rio de Janeiro: Instituto Brasileiro de Geografia e Estatística; 2009. (Estudos \& Pesquisas. Informação Demográfica e Socioeconômica, 25).

25. Kessner DM, Kalk CE, Singer J. Assessing health quality-the case for tracers. N Engl J Med 1973; 288:189-94.

26. Landis JR, Koch GG. The measurement of observer agreement for categorical data. Biometrics 1977; 33:159-72.

27. Donabedian A, Wheeler HRC, Wyszewianski L. Quality, cost and health: an integrative model. Med Care 1982; 20:1975-92.

28. Miranda AE, Trindade CR, Nunes RH, Marba EF Fernandes MC, Quarto GHA, et al. Factors associated with prenatal care and seeking assistance in public hospitals in Vitória, Espírito Santo, Brazil. Women Health 2010; 50:229-40.

29. Bassani DG, Surkan PJ, Olinto MT. Inadequate use of prenatal services among Brazilian women: the role of maternal characteristics. Int Perspect Sex Reprod Health 2009; 35:15-20.

30. Chrestani MAD, Santos IS, Cesar JA, Winckler L, Gonçalves TS, Neumann NA. Assistência à gestação e ao parto: resultados de dois estudos transversais em áreas pobres das regiões Norte e Nordeste do Brasil. Cad Saúde Pública 2008; 24:1609-18.

31. Grangeiro GR, Diógenes MAR, Moura ERF. Atenção pré-natal no município de Quixadá-CE segundo indicadores de Sisprenatal. Rev Esc Enferm USP 2008; 42:105-11. 
32. Andreucci CB, Cecatti JG, Macchetti CA, Sousa MH. Sisprenatal como instrumento de avaliação da qualidade da assistência à gestante. Rev Saúde Pública 2011; 45:854-63.

33. Domingues RMSM, Hartz ZMA, Dias MAB, Leal MC. Avaliação da adequação da assistência prénatal na rede do SUS do município do Rio de Janeiro, Brasil. Cad Saúde Pública 2012; 28:425-37.

34. Parada CMGL. Avaliação da assistência pré-natal e puerperal desenvolvidas em região do interior do Estado de São Paulo em 2005. Rev Bras Saúde Matern Infant 2008; 8:113-24.
35. Barreto FDFP, Albuquerque RM. Discrepâncias entre o informe verbal e os registros no cartão da gestante, um instrumento negligenciado. Rev Bras Ginecol Obstet 2012; 34:259-67.

36. Almeida SD M, Barros MBA. Equidade e atenção à saúde da gestante em Campinas (SP), Brasil. Rev Panam Salud Pública 2005; 17:15-25.

Recebido em 27/Set/2012

Versão final reapresentada em 17/Dez/2012

Aprovado em 08/Jan/2013 